\title{
Correlation between palpatory assessment and pressure sensors in response to postural trunk tests
}

\author{
Jakub Jacisko ${ }^{\mathrm{a}, *}$, Martin Stribrny ${ }^{\mathrm{a}}$, Jakub Novak ${ }^{\mathrm{a}}$, Andrew Busch ${ }^{\mathrm{b}}$, Pavel Cerny ${ }^{\mathrm{c}}$, Pavel Kolar ${ }^{\mathrm{a}}$ and \\ Alena Kobesova ${ }^{a}$ \\ ${ }^{a}$ Department of Rehabilitation and Sports Medicine, Second Faculty of Medicine, Charles University and University \\ Hospital Motol, Prague, Czech Republic \\ ${ }^{\mathrm{b}}$ Department of Health and Human Kinetics, Ohio Wesleyan University, Delaware, OH, USA \\ ${ }^{\mathrm{c}}$ Faculty of Health Care Studies, University of West Bohemia, Plzen, Czech Republic
}

Received 10 October 2020

Accepted 12 December 2020

\begin{abstract}
.
BACKGROUND: The evaluation of postural trunk muscle function is a critical component of clinical assessment in patients with musculoskeletal pain and dysfunction. Postural activation of the trunk muscles has been evaluated by various methods. This study evaluates the correlation between subjective assessment of postural trunk muscle function with an objective measurement of abdominal wall expansion.

METHODS: Twenty-five healthy participants (16 women, 9 men, age 22.4 years) were assessed. The subjective assessment was performed by two experienced Dynamic Neuromuscular Stabilization (DNS) clinicians evaluating the quality of trunk stabilization using five postural stability tests through palpation and observation. Interrater reliability was determined using an intraclass correlation coefficients (ICC). Objective measurement was performed using a new device (DNS Brace) which externally measures abdominal wall pressure. Spearman rank correlations were calculated for both palpation and observation measures with DNS Brace data.

RESULTS: The interrater reliability (ICC2,k) estimates demonstrated moderate reliability in palpation measures for three DNS tests: Hip flexion test, Diaphragm test, \& Intra-abdominal pressure regulation test (IAPRT) (ICC $=0.645-0.707$ ). For observation measures, good reliability was found in IAPRT $(\mathrm{ICC}=0.835)$, and three tests demonstrated moderate reliability: Hip flexion test, Diaphragm test, \& Breathing Stereotype (ICC $=0.577-0.695)$. Correlation analysis demonstrated several moderate to strong correlations between palpation and DNS brace values (Assessor 1): IAPRT, $r_{s}=0.580, p=0.002$, Diaphragm test, $r_{s}=0.543$, $p=0.005$, (Assessor 2): IAPRT, $r_{s}=0.776, p<0.001$, Breathing Stereotype, $r_{s}=0.625, p=0.001$, Diaphragm test, $r_{s}=$ $0.519, p=0.008$, Hip Flexion test, $r_{s}=0.536, p=0.006$, and Arm Elevation test, $r_{s}=0.460, p=0.021$. For observation, several moderate correlations were demonstrated with DNS brace values (Assessor 1): Arm Elevation test, $r_{s}=0.472, p=0.017$, (Assessor 2) Diaphragm test, $r_{s}=0.540, p=0.005$, IAPRT $r_{s}=0.475, p=0.016$, Hip Flexion test, $r_{s}=0.485, p=0.014$, and Arm Elevation, $r_{s}=0.451, p=0.024$.

CONCLUSION: Based on inter-rater reliability and DNS brace correlations with trained DNS professionals, the IAPRT, Diaphragm test, and Hip Flexion test may prove useful when assessing asymptomatic individuals. More research is needed in order to establish the utility of DNS brace and clinical testing both in asymptomatic and back pain populations. DNS tests must be supplemented by further examinations for definitive clinical decision making.
\end{abstract}

Keywords: Postural stabilization, breathing, abdominal muscles, intra-abdominal pressure, Dynamic Neuromuscular Stabilization

${ }^{*}$ Corresponding author: Jakub Jacisko, Department of Rehabilitation and Sports Medicine, Second Faculty of Medicine, Charles University and University Hospital Motol, V Úvalu 84150 06, Prague,
Czech Republic. Tel.: +420 776246648; E-mail: jakub.jacisko@ gmail.com. 


\section{Introduction}

Evaluation of postural trunk muscle function is a critical component of clinical assessment in patients with musculoskeletal pain and dysfunction. Activation of the postural trunk muscles is essential for maintaining IAP (Intra-Abdominal Pressure) [1,2]. Appropriate IAP regulation secures stability of the lumbar spine $[3,4]$. Altered function of trunk muscles is associated with low back pain (LBP) [5,6] which is a major public health problem worldwide causing significant personal and financial burden [7]. Numerous studies suggest that trunk and lumbar spine stabilization exercises may help in LBP treatment and contribute to LBP prevention [8].

Postural activation of the trunk muscles has been evaluated by various methods such as ultrasonography [9], electromyography [9], pressure biofeedback unit [10], dynamometry [11] or direct IAP measurement $[3,4]$. Although some of these methods can measure the core activity or even IAP quite accurately, most of them serve for research purpose rather than clinical practice because the procedures may be uncomfortable for the patient, invasive, time consuming and often difficult to interpret the results. In routine clinical practice subjective assessment via various clinical tests is the most common way to evaluate postural function of the trunk muscles [12,13].

One concept offering a complete set of clinical tests to evaluate closely inter-related postural-respiratory functions [14] is Dynamic Neuromuscular Stabilization (DNS) [13]. DNS is a functional diagnostic and therapeutic approach based on human ontogenesis applying principles of movement and posture development during the first years of a healthy individual's life $[15,16]$. The complete set of DNS testing [13] captures the stereotype of postural stabilization and movement [17], respiratory pattern $[14,18]$, functional joint centration [15] and segmental movement [19], while offering a functional treatment plan for musculoskeletal [20,21] or neurological patients [22]. Still, there is a need for more objective data to demonstrate the reliability of DNS procedures and to monitor the progress or improvements based on DNS principles.

Therefore, this study presents a new, non-invasive device called DNS Brace which objectively measures the expansion of the abdominal wall, a function which purportedly correlates with IAP changes and breathing [1]. Expansion of the abdominal wall related to IAP regulation is an important mechanism of trunk and spinal stabilization [14]. Additionally, this study examines the correlation between a clinician's subjective postural function assessment and objective measurement of the abdominal wall expansion using the DNS Brace.

\section{Methods}

\subsection{Participants}

The study was approved by the local ethics committee (Protocol number 17954, 8.1. 2020, Ethics committee of the Second Faculty of Medicine, Charles University and University Hospital Motol, Prague, Czech Republic). Participants were addressed via social media. Exclusion criteria were any symptomatic neurologic, orthopedic, respiratory or musculoskeletal disorder, spine or abdominal surgery, severe trauma during the last year, pregnancy, and undergoing DNS therapy in the past. In total 25 participants, 16 women and 9 men were involved in the study. Before the assessment, every participant received the same detailed information about the testing procedure. Every participant signed the informed consent. Basic descriptive data including gender, age, anthropometric data were recorded for each participant. Table 1 shows general characteristics for the whole group.

\subsection{Instrumentation}

The DNS Brace (Produced by Ortotika, FN Motol V Úvalu 84, Praha, (Fig. 1) which is mechanically configured as a trunk orthosis is equipped with four sensors working on a mechanical-pneumatic-electronic principle. For assessment, the brace fits tightly to bony structures allowing the expansion of soft tissues. The sensors are fixed on the inner wall of the brace. Two sensors are located on the brace parts adhering to laterodorsal sections (trigonum lumbale) of the abdominal wall and two are placed above the groin. The position of the sensors can be easily adjusted to fit each individual. The sensor heads are hemispheric in shape, allowing them to adhere to soft tissues in the monitored locations. Each sensor head contains an inner-air chamber and is made from silicone, which provides stable mechanical quality in a wide range of temperatures. The inner-air chamber is connected to a digital pressure sensor via a capillary tube. When recording measurements, each sensor's silicone head is deformed by the applied pressure, which causes a reduction of volume in the inner-air chamber, thus increasing pneumatic pressure in the inner capillary system. Pneumatic pressure is registered via an 
Table 1

Participant's anthropometric characteristics. $n=25,9$ males, 16 females

\begin{tabular}{lccccc}
\hline & Age (year) & Height $(\mathrm{m})$ & Weight $(\mathrm{kg})$ & BMI $\left(\mathrm{kg} / \mathrm{m}^{2}\right)$ & Waist $(\mathrm{cm})$ \\
\hline Mean & 22.4 & 172.68 & 68.88 & 23.02 & 77.16 \\
SD & 1.76 & 7.26 & 8.66 & 1.58 & 5.98 \\
Min & 20 & 161 & 58 & 19.82 & 67 \\
Max & 25 & 190 & 85 & 25.85 & 90 \\
\hline
\end{tabular}

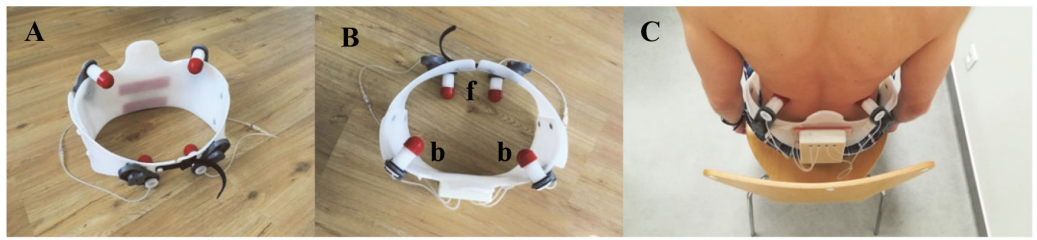

Fig. 1. DNS brace A. Front view; B. Top view, $\mathrm{f}$ - two sensors in the front, $\mathrm{b}$ - two sensors in the back; C. Back view, Brace on a man.

Deep breathing

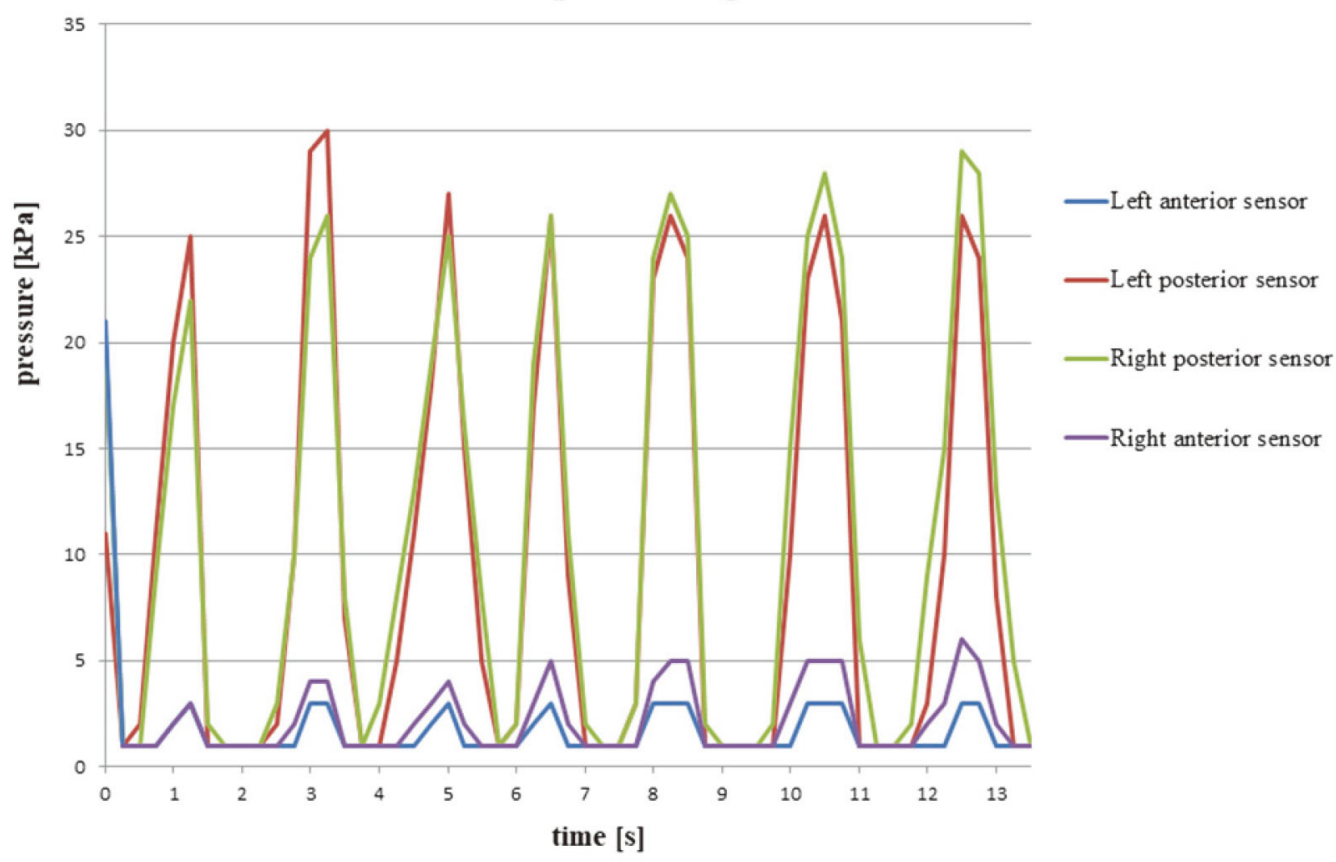

Fig. 2. Data from DNS Brace measuring deep breathing displayed in a graph.

auxiliary electric device (digital pressure sensor) on the orthosis. The sensors register the pressure exerted by the abdominal wall. The values recorded in kilopascals $(\mathrm{kPa})$ are transferred via Bluetooth, stored and graphically displayed in a smart-phone device (Fig. 2). The sampling rate is $240 \mathrm{~Hz}$.

\subsection{Procedure}

Participants ( $n=25$ ) were randomly assigned to two groups. Participants from group 1 ( $n=13$, female 8 , male 5) were first assessed by the two DNS assessors in a random order (some participants were first assessed by assessor \#1 and then by assessor \#2 or vice versa), and subsequently by DNS Brace which was applied by another clinician. Participants from group $2(n=12$, female 8 , male 4) were first assessed by DNS Brace, and subsequently by the two DNS assessors in random order. The measurements were always performed under the same environmental conditions.

DNS assessors evaluated the five postural tests according to DNS (as described below) consecutively in 


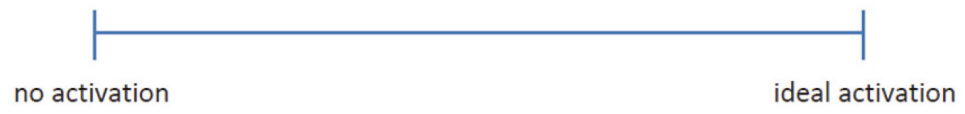

Fig. 3. Visual-analogue scale for subjective assessment of postural tests (the assessors made two lines on VAS - one for aspection, one for palpation).

the same order on each participant. Every participant was given exactly the same instructions before each test. After assessing all 5 tests by the first DNS assessor, the participant was assessed by the other DNS assessor, who gave the same instructions to evaluate each test. There was no time limit for the evaluation. Both assessors assessed each participant first by palpation and then visually using VAS (visual analogue scale) from 0 (no activation) to 100 (ideal activation) [23] (Fig. 3). Palpation by DNS assessors was performed at the same body regions where the DNS Brace sensors were placed, i.e. in trigonum lumbale bilaterally and above the groin bilaterally. DNS tests were reported as reliable assessment methods in other research projects previously [24].

\subsection{Subjective assessment}

Five DNS postural tests were performed by each participant and evaluated by two experienced assessors (certified DNS instructors) by palpation and inspection using VAS from 0 to 100 points where 0 represents absolute inability to perform required activity and 100 represents ideal activation (Fig. 3).

During all five tests the participants were seated, their hips and knees flexed in $90^{\circ}$ angle, feet touching the ground while keeping spine upright and shoulders relaxed. With each participant the tests were evaluated in the following order:

1. Breathing stereotype test. (Fig. 4) The participant was instructed to take five deep breaths. The assessor first palpated the activation in the lower intercostal spaces and below the lower ribs bilaterally and then above the groin. Then, the assessor performed visual observation focusing on lower ribs and shoulder movement.

2. Intra-abdominal pressure regulation test. (Fig. 5) The assessor palpated bilaterally the lower abdominal sections above the groin. The participant was instructed to activate the IAP by pushing against assessor's fingers. Amount and symmetry of activation is assessed by palpation. Then, visual observation of abdominal contour, umbilicus and shoulder movement was performed.

3. The diaphragm test. (Fig. 6) The assessor was po-

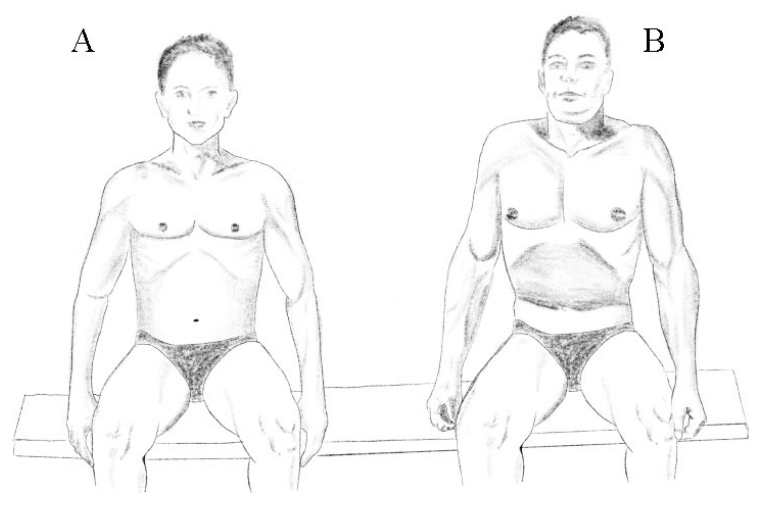

Fig. 4. Breathing stereotype test. A. Optimal pattern. Spine upright, trunk in neutral position, relaxed auxiliary breathing muscles, proportional expansion of abdominal wall occurs with inhalation. B. Pathological stereotype. The chest moves superiorly, shoulders moves superiorly and into protraction during inhalation, insufficient or no expansion of the abdominal wall.

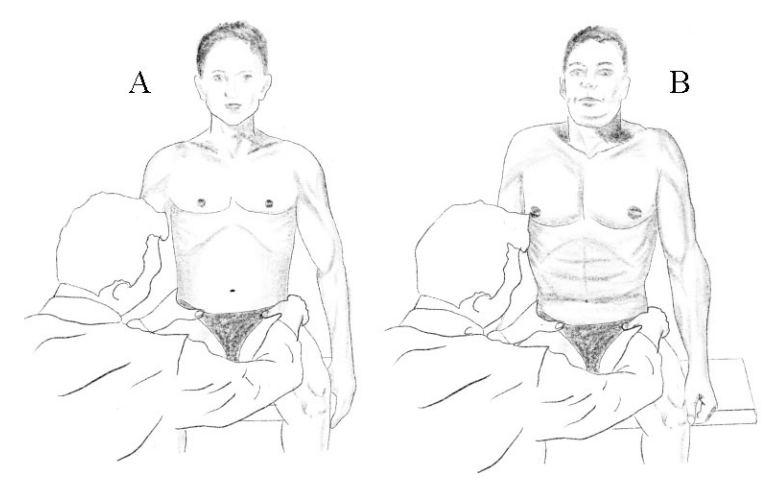

Fig. 5. Intra-abdominal pressure regulation test. A. Optimal pattern. Proportional tensing of abdominal wall in all sections. B. Pathological stereotype. Inability to expand lower abdominal wall, asymmetrical activation, overactivity of upper rectus abdominis muscle, ribcage elevation.

sitioned behind the participant palpating bilaterally below participant's lower ribs. The participant was instructed to take a deep breath and push towards assessor's fingers to activate the abdominal wall. The assessor evaluated the amount and symmetry of activation of the abdominal wall. Then, the assessor visually observed lateral movement of the lower ribs while monitoring the spine (upright and stable) and the presence of shoulder movement or pathological synkinesis (Fig. 6). 

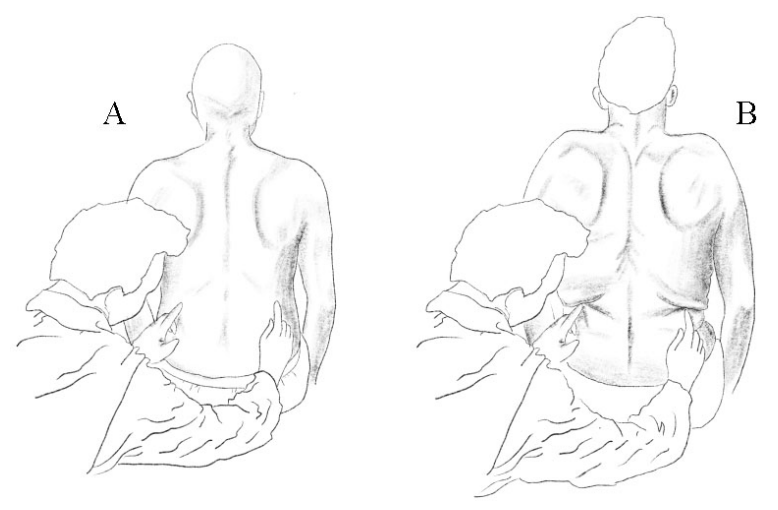

Fig. 6. Diaphragm test. A. Optimal pattern. Abdominal wall eccentric expansion, upright spine, without shoulder movements cranially. B. Pathological stereotype. Inability to expand latero-dorsal parts of the abdominal wall, asymmetrical activation, rib cage or shoulder elevation, substitutive mechanism with spinal kyphosis compensating for lack of eccentric abdominal wall activation.
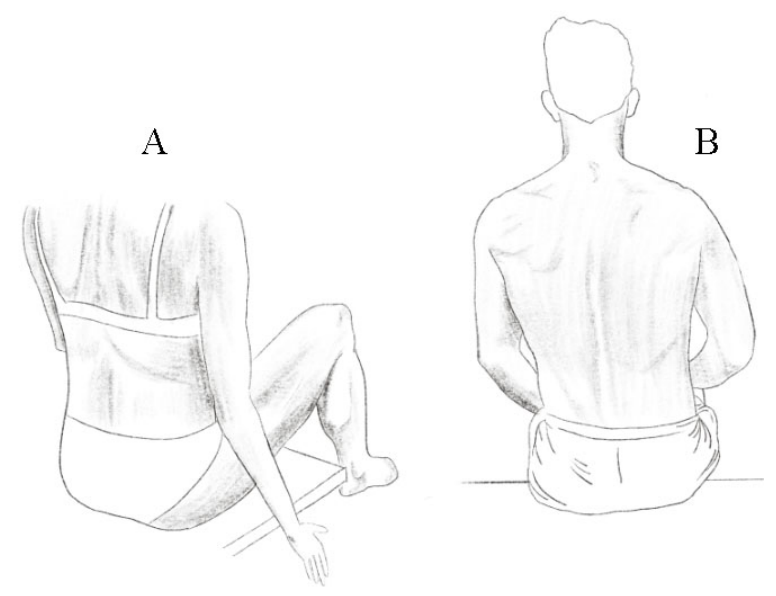

Fig. 7. Hip flexion test. A. Optimal pattern. Chest and pelvis in neutral position, spine upright. B. Pathological stereotype. Inability to keep the spine upright and pelvis stable, lateral shift of the trunk.

4. Hip flexion test. (Fig. 7) The assessor instructed the participant to slowly lift up right leg (approximately 10 to $20 \mathrm{~cm}$ ) above the ground. Participant breathed naturally while maintaining this position. The activity of the latero-dorsal sections of abdominal wall was assessed bilaterally by palpation (as in diaphragm test). Then, any spinal and pelvic movements were assessed by visual inspection.

5. Arm lifting test. (Fig. 8) The participant lifted a dumbbell that corresponded to $20 \%$ of the body weight. Elbows were flexed to $90^{\circ}$ and participant breathed naturally in this position. The assessor palpated bilaterally the abdominal wall activation first in trigonum lumbale, then above the groin.

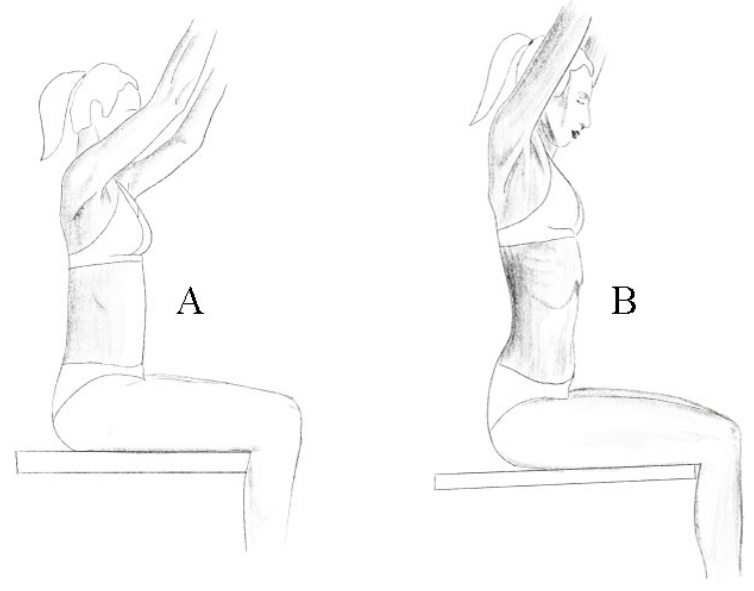

Fig. 8. Arm lifting test. A. Optimal pattern. Ribcage in neutral position, thoracolumbar junction stable, symmetrical expansion of abdominal wall. B. Pathological stereotype. Chest elevation, thoracolumbar instability, hyperlordosis of the lumbar spine.

Spinal or pelvic movements were assessed visually.)

All DNS tests were performed according to the detailed procedures described by Kobesova et al. 2020, which include all signs of optimal and abnormal presentations [13].

\subsection{DNS Brace measurements}

The DNS Brace was applied by another clinician and was attached around the participant's trunk. The activity of the abdominal wall was monitored during the same five DNS tests and in the same order as with the subjective assessment. The same instructions were given for DNS Brace measurement as for the subjective testing. The sensors were always calibrated to $0 \mathrm{kPa}$ in resting exhalation position prior to each measurement. Afterwards the participants were instructed to take two natural breaths and then the test was performed and the abdominal wall activity recorded. A total of 3 to 7 breathing cycles were recorded (recording time was between 6 to 20 seconds depending on individual breathing speed). Afterwards, the average pressure against all four sensors was calculated and used for correlation with the subjective clinical assessment. The assessors were not allowed to view results received from the DNS Brace measurements nor were they allowed to consult each other during testing.

\subsection{Statistical analysis}

Descriptive data were calculated, including means 
Table 2

Interrater reliability of different DNS tests using palpation and observation $\left(\mathrm{ICC}_{2, \mathrm{k}}\right)$

\begin{tabular}{|c|c|c|c|c|c|c|}
\hline \multirow[b]{2}{*}{ DNS test } & \multicolumn{3}{|c|}{ Palpation } & \multicolumn{3}{|c|}{ Observation } \\
\hline & ICC & $95 \% \mathrm{CI}$ & Sig & ICC & $95 \% \mathrm{CI}$ & Sig \\
\hline 1-Breathing stereotype & 0.446 & $(-0.258,0.756)$ & 0.078 & $0.695^{*}$ & $(0.308,0.866)$ & 0.003 \\
\hline 2-IAPRT & $0.707^{*}$ & $(0.334,0.871)$ & 0.002 & $0.835^{* *}$ & $(0.626,0.927)$ & 0.000 \\
\hline 3-Diaphragm test & $0.646^{*}$ & $(0.197,0.844)$ & 0.007 & $0.668^{*}$ & $(0.246,0.854)$ & 0.005 \\
\hline 4-Hip flexion test & $0.645^{*}$ & $(0.194,0.843)$ & 0.007 & $0.577^{*}$ & $(0.04,0.814)$ & 0.020 \\
\hline 5-Arm elevation & 0.308 & $(-0.570,0.695)$ & 0.187 & 0.464 & $(-0.217,0.764)$ & 0.067 \\
\hline
\end{tabular}

Note: DNS = Dynamic neuromuscular stabilization; ICC = Intraclass correlation coefficient; IAP = Intra abdominal pressure regulation test; Both examiners were trained DNS professionals. ${ }^{*}$ Denotes: Moderate reliability. ${ }^{* *}$ Denotes: Good reliability.

Table 3

Correlations of DNS instructor values with average DNS brace values in five DNS tests (mean [standard deviation])

\begin{tabular}{|c|c|c|c|c|c|c|c|}
\hline \multirow[b]{2}{*}{ DNS test } & \multirow[b]{2}{*}{ DNS brace average values } & \multicolumn{3}{|c|}{ Palpation } & \multicolumn{3}{|c|}{ Observation } \\
\hline & & Score & Spearman $r_{s}$ & Sig & Score & Spearman $r_{s}$ & Sig \\
\hline \multicolumn{8}{|l|}{ DNS Assessor 1} \\
\hline 1-Breathing stereotype & $4.89(3.18)$ & $70.28(19.43)$ & 0.443 & 0.026 & $65.56(20.85)$ & 0.290 & 0.159 \\
\hline 2-IAPRT & $12.19(8.47)$ & $84.12(14.27)$ & 0.580 & $0.002^{*}$ & $76.88(16.25)$ & 0.380 & 0.061 \\
\hline 3-Diaphragm test & $11.73(9.11)$ & $76.16(17.06)$ & 0.543 & $0.005^{*}$ & $71.32(16.86)$ & -0.105 & 0.616 \\
\hline 4-Hip flexion test & $6.28(5.52)$ & $65.44(19.53)$ & 0.338 & $0.009^{*}$ & $70.20(17.34)$ & -0.039 & 0.852 \\
\hline 5-Arm elevation & $9.44(8.80)$ & $77.12(14.12)$ & 0.303 & 0.142 & $75.80(10.91)$ & 0.472 & $0.017^{*}$ \\
\hline \multicolumn{8}{|l|}{ DNS Assessor 2} \\
\hline 1-Breathing stereotype & $4.89(3.18)$ & $43.60(13.32)$ & 0.625 & $0.001^{*}$ & $41.68(15.87)$ & 0.342 & 0.094 \\
\hline 2-IAPRT & $12.19(8.47)$ & $53.40(20.58)$ & 0.776 & $<0.001^{*}$ & $47.60(19.35)$ & 0.475 & $0.016^{*}$ \\
\hline 3-Diaphragm test & $11.73(9.11)$ & $52.40(16.83)$ & 0.519 & $0.008^{*}$ & $49.64(21.75)$ & 0.540 & $0.005^{*}$ \\
\hline 4-Hip flexion test & $6.28(5.52)$ & $47.20(13.00)$ & 0.536 & $0.006^{*}$ & $45.68(15.23)$ & 0.485 & $0.014^{*}$ \\
\hline 5-Arm elevation & $9.44(8.80)$ & $46.08(15.79)$ & 0.460 & $0.021^{*}$ & $45.68(20.43)$ & 0.451 & $0.024^{*}$ \\
\hline
\end{tabular}

Note: DNS = Dynamic neuromuscular stabilization; IAPRT = Intra-abdominal pressure regulation test; Both examiners were trained DNS professionals. * Statistically significant correlation (Bonferroni Correction $P<0.025$ ).

and standard deviations (SD) for each DNS assessor's palpation and observation using the VAS, and the DNS brace values. Interrater reliability was determined using intraclass correlation coefficients $\left(\mathrm{ICC}_{2, \mathrm{k}}\right)$ and their 95\% confidence intervals $(\mathrm{CI})$ between the two DNS assessors' measures of either palpation or observation for all five DNS tests based on a mean-rating $(k=2)$, consistency, 2-way random model. Reliability was defined as poor (ICC $<0.50$ ), moderate (ICC $0.50-0.75$ ), or good (ICC > 0.75). Spearman's rank order correlations were used to analyze the relationship between different DNS assessors measures with the average DNS Brace values. The strength of correlations were interpreted as weak $(<0.3)$, moderate $(0.4-0.6)$, or strong $(>0.7)$, as reported by Akoglu [25]. The alpha level used for significance, with Bonferroni corrections, was set a priori at $p<0.025$. All data was analyzed using SPSS statistical package v26 (SPSS Inc, Chicago, IL).

\section{Results}

Descriptive data for all participants are presented in Table 1 . Not all variables were normally distributed, as assessed by Shapiro-Wilk's test $(p<0.05)$. Interrater reliability ICC estimates are shown in Table 2. For palpation, moderate reliability was demonstrated duirng three DNS tests: Hip flexion test, Diaphragm test, \& IAPRT (ICC $=0.645-0.707$ ). For observation, moderate reliability was again demonstrated in three tests: Hip flexion test, Diaphragm test, \& Breathing Stereotype (ICC $=0.577-0.695)$ while a good reliability was found in IAPRT (ICC $=0.835)$.

All correlational data with $95 \%$ confidence intervals are presented in Table 3. For DNS Assessor 1, palpation demonstrated moderate correlations between the DNS brace values with IAPRT, $r_{s}(23)=0.580, p=$ 0.002 and the Diaphragm test, $r_{s}(23)=0.543, p=$ 0.005 while observation demonstrated a lower correlation with the Arm Elevation test, $r_{s}(23)=0.472, p=$ 0.017. For DNS instructor 2, palpation demonstrated a strong correlation for for IAPRT, $r_{s}(23)=0.776$, $p<0.001$ and modetate correlations for the Breathing Stereotype, $r_{s}(23)=0.625, p=0.001$, Diaphragm test, $r_{s}(23)=0.519, p=0.008$, and Hip Flexion test, $r_{s}(23)=0.536, p=0.006$. A lower correlation was demonstrated with the Arm Elevation test, $r_{s}(23)=$ 0.460, $p=0.021$. Observation for DNS Assessor 2 
demonstrated moderate correlations in the Diaphragm test, $r_{s}(23)=0.540, p=0.005$, IAPRT $r_{s}(23)=$ $0.475, p=0.016$, Hip Flexion test, $r_{s}(23)=0.485$, $p=0.014$, and Arm Elevation, $r_{s}(23)=0.451, p=$ 0.024 .

\section{Discussion}

Trunk stabilization analysis is critical part of clinical assessment. Postural function is closely related to movement and locomotion; mobility and stability form a functional unit that is under the constant control of central nervous system [26]. Another function closely related to trunk stabilization is respiration. The respiratory stereotype affects trunk muscle coordination and modifies the movement [27] therefore specific breathing instructions form a critical part of many stabilization exercise protocols [28,29]. Some studies indicate, that impaired postural control is associated with chronic low back pain [30]. Trunk stabilization training is often applied to treat and prevent back pain and other musculoskeletal problems and injuries [31].

The first step to analyze the influence of postural stabilization training on movement performance and musculoskeletal pain is to define the optimal pattern of postural stabilization. Due to extreme postural variability the exact definition of optimal posture is still ambiguous with each author defining the ideal posture differently [32-34]. One concept that aims to define optimal postural stabilization is Dynamic Neuromuscular Stabilization [35]. This DNS concept derives an ideal stabilization stereotype from genetically predetermined developmental patterns observed during normal early human ontogenesis. DNS offers a set of a functional diagnostic tests and evaluation to monitor a patient's posture [13]. However, the reliability of DNS clinical tests has not been demonstrated yet. This study correlates subjective assessment via five DNS tests performed by two experienced DNS clinicians with objective measurement of abdominal wall activity using new device called DNS Brace. The correlation between the subjective DNS assessments and objective measurement of abdominal wall expansion may help to determine the reliability of clinical DNS tests. At the same time this study reports interrater reliability for the five DNS tests. Additionally, this study introduces a new, simple and non-invasive device to measure the activity of the abdominal wall.

For both palpation $(\mathrm{ICC}=0.707)$ and observation $(\mathrm{ICC}=0.835)$ assessments, the IAPRT test demon- strated the best reliability between assessors. We identified moderate interrater reliability for both palpation and observation for the Diaphragm and the Hip Flexion test. Considering the complexity of DNS assessment, which emphasizes much detail and nuance both in palpation and observation assessment, the findings of moderate-good ICC's for 3/5 DNS tests was encourraging. These findings are similar to other well established systems, such as Mechanical Diagnosis and Therapy (MDT), where reported inter-rater reliability ranges from 0.11 to 1.00 [36]. Much more reasearch is needed to establish the relevance of DNS testing both in normal cohorts and in populations with various musculoskeletal problems.

The results of this study confirmed a positive correlation between objectively measured expansion of the abdominal wall and subjective palpatory assessment of postural trunk muscle function according to the DNS approach. For assessor 1, statistically significant correlation was identified for three DNS tests (IAPRT, Diaphragm and Hip Flexion test) and for assessor 2, palpation significantly correlated with all 5 DNS tests Brace measurements. The increase in pressure against the sensors was the highest in the situations when the measured participant was instructed to activate the IAP, i.e. when he or she had to push specifically against the sensors above the groin (IAPRT; average pressure $=12.19 \mathrm{kPa}$ ) or against the sensors placed in trigonum lumbale (diaphragm test; average pressure $=11.73 \mathrm{kPa}$ ). The third highest value was recorded during the arm elevation test holding the weight (average pressure $=9.44 \mathrm{kPa}$ ). The hip flexion test required $6.28 \mathrm{kPa}$ average pressure increase only, yet such change was appropriately recognized by palpating assessors. During all these tests positive correlation between subjective and objective assessment was confirmed. The only exception was the breathing stereotype test where significant correlation between objective DNS Brace testing and subjective palpatory assessment was reached in one assessor only (see Table 2). During the breathing stereotype test the lowest average pressure increase $(4.89 \mathrm{kPa})$ was measured by DNS Brace. It can be assumed that the smaller the change in the activation, the more difficult it is to estimate the amount of change by mere palpation.

Based on these results, instructed activation tests such as IAPRT or the Diaphragm test appear potentially useful in evaluating trunk stabilization function in clinical practice. Still, such tests need to be supplemented by further examinations for definitive clinical decision making. Surprisingly, lifting the weight corresponding to $20 \%$ of the participant's body weight evoked less pos- 
tural stabilization activity than an instructed increase in IAPRT. Apparently, in healthy individuals such weight does not require much activity of the abdominal wall. This result further supports the study published by Essendrop et al. who report IAP increase from 0 to $40 \%$ when holding a load of $15 \%$ body weight [4].

The DNS Brace measures both voluntarily controlled and automatic subconscious postural activation. We can either instruct the individual to activate their abdominal wall pushing against the sensors, thus the brace can also be used for feedback training or we can monitor spontaneous level of activation during various movements. Both situations may be convenient in clinical practice and in research. Advantages of using the DNS Brace lie in it's fixed position of all 4 sensors that maintains contact with the trunk allowing the assessment in various positions and when moving. Such modifications may not be available when using other devices designed to measure and train abdominal muscles and lumbopelvic stability such as a Pressure Biofeedback Unit [37] or ultrasound which analyze mainly local activation of the abdominal muscles. The information from the four DNS Brace sensors is a more global monitoring of abdominal muscle co-activation. Also, it is very easy to apply the DNS Brace and to record and analyze results. Unlike electromyography, ultrasound or direct IAP measurement techniques, there is no need for special personnel training.

The entire assessment took approximately 8 minutes with each assessor, i.e. 24 minutes all together (2 subjective assessments + DNS brace assessments). The measurement order between assessor 1, 2 and DNS Brace was random to exclude the influence of any motor learning. The participants considered the examination to be both physically and mentally easy. The DNS Brace measurement starts from a fully relaxed state that does not require any pre-tensioning. In a study using a Pressure Biofeedback Unit, participants were instructed to maintain the target pressure range ( $40 \pm$ $10 \mathrm{mmHg}$ ) [38]. This may exclude some individuals who are unable to reach such starting pressure. The DNS brace does not require any minimum prerequisite pressure to start the measurement, making the procedure simple, convenient and clinically useful. The DNS Brace measurement range is 0 to $500 \mathrm{kPa}$. The values measured by the brace are absolute thus comparable in time or among raters.

Correlation and statistical significance for palpation was in most cases better than that for observation. Palpation is an integral skill forming the vital component of many hands-on clinical examinations [39] including
DNS concept. The results of this study support the use of the DNS tests described above when performed by skilled DNS clinicians. In this study the tests were evaluated by experienced DNS clinicians with more than five years clinical experience. This is an important aspect to consider since the palpation accuracy is closely related to examiner's experience and training [40]. To our knowledge this is the first study correlating subjective assessment of postural function of the trunk muscles with objective measurement of the abdominal wall expansion. The inspection should rather be complementary to palpation.

Future studies should investigate the correlation between abdominal wall expansion and direct IAP measurement to find out if DNS brace can actually replace invasive and uncomfortable techniques of direct IAP monitoring such as intravesical, anal or vaginal pressure measurements. Also, inter- and intra-rater reliability for DNS Brace needs to be established. Finally, the relationship between LBP and abdominal wall expansion needs to be explored as well as the effect of abdominal wall training. In the future DNS Brace may become a useful clinical and research tool.

There are some limitations to this study. First, this study was done in asymptomatic individuals. It is unknown if such DNS tests would show similar results in LBP patients or in individuals with other musculoskeletal disorders. Future studies in patients with LBP are warranted. Second, the average pressure against all four sensors was calculated and used for statistical analysis and the symmetry of the abdominal wall expansion was not considered. The brace could not be properly used in one extremely slim individual who had to be excluded from the study. In the future, smaller versions of DNS Braces will be constructed. Finally, DNS tests were assessed by DNS professionals (DNS instructors) with more than 5 years DNS experience. Future research could compare novice pracitioners with experienced, to learn differences.

\section{Conclusion}

Based on interrater reliability and DNS brace correlations with trained DNS professionals, the IAPRT, Diaphragm test, and Hip Flexion test may be useful for clinicians when assessing normal individuals. More research is needed in order to establish the utility of DNS brace and DNS clinical testing both in normal and back pain populations. 


\section{Acknowledgments}

The authors have no acknowledgments.

\section{Author contributions}

CONCEPTION: Jakub Jacisko, Jakub Novák, Martin Stribrny, Alena Kobesova, Andrew Busch, Pavel Cerny and Pavel Kolar.

PERFORMANCE OF WORK: Jakub Jacisko, Martin Stribrny, Jakub Novak and Pavel Cerny. INTERPRETATION OR ANALYSIS OF DATA: Andrew Busch, Jakub Jacisko, Alena Kobesova, Martin Stribrny and Pavel Cerny.

PREPARATION OF THE MANUSCRIPT: Jakub Jacsiko, Alena Kobesova, Andrew Busch and Pavel Cerny. REVISION FOR IMPORTANT INTELLECTUAL CONTENT: Alena Kobesova and Andrew Busch. SUPERVISION: Alena Kobesova, Andrew Busch, Pavel Cerny, Jakub Jacisko and Pavel Kolar.

\section{Ethical considerations}

The study was approved by the local ethics committee (Protocol number 17954, 8.1. 2020, Ethics committee of the Second Faculty of Medicine, Charles University and University Hospital Motol, Prague, Czech Republic). Before the assessment, every participant received the same detailed information about the testing procedure. Every participant signed the informed consent.

\section{Conflict of interest}

The authors have no conflict of interest to declare.

\section{Funding}

This study was supported by Institutional research program Progres Q41 and by by the foundation Movement Without Help.

\section{References}

[1] De Troyer A, Boriek AM. Mechanics of the Respiratory Muscles. In: Terjung R, ed. Comprehensive Physiology [Internet].
Hoboken, NJ, USA: John Wiley \& Sons, Inc.; 2011 [cited 2020 Jul 4]. p. c100009. Available from: doi: 10.1002/cphy.c100009.

[2] Hodges PW, Gandevia SC. Changes in intra-abdominal pressure during postural and respiratory activation of the human diaphragm. J Appl Physiol. 2000 Sep 1; 89(3): 967-76.

[3] Stokes IAF, Gardner-Morse MG, Henry SM. Abdominal muscle activation increases lumbar spinal stability: analysis of contributions of different muscle groups. Clin Biomech. $2011 \mathrm{Oct}$; 26(8): 797-803

[4] Essendrop M, Andersen TB, Schibye B. Increase in spinal stability obtained at levels of intra-abdominal pressure and back muscle activity realistic to work situations. Appl Ergon. 2002 Sep; 33(5): 471-6

[5] Hemming R, Sheeran L, van Deursen R, Sparkes V. Investigating differences in trunk muscle activity in non-specific chronic low back pain subgroups and no-low back pain controls during functional tasks: a case-control study. BMC Musculoskelet Disord. 2019 Dec; 20(1): 459.

[6] Lin Y-C, Niu C-C, Nikkhoo M, Lu M-L, Chen W-C, Fu C-J, et al. Postural stability and trunk muscle responses to the static and perturbed balance tasks in individuals with and without symptomatic degenerative lumbar disease. Gait Posture. 2018 Jul; 64: 159-64.

[7] Hoy D, Bain C, Williams G, March L, Brooks P, Blyth F, et al. A systematic review of the global prevalence of low back pain. Arthritis Rheum. 2012 Jun; 64(6): 2028-37.

[8] Horsak B, Wunsch R, Bernhart P, Gorgas A-M, Bichler R, Lampel K. Trunk muscle activation levels during eight stabilization exercises used in the functional kinetics concept: a controlled laboratory study. J Back Musculoskelet Rehabil. 2017 May 5; 30(3): 497-508.

[9] Brown SHM, McGill SM. A comparison of ultrasound and electromyography measures of force and activation to examine the mechanics of abdominal wall contraction. Clin Biomech. 2010 Feb; 25(2): 115-23.

[10] von Garnier K, Köveker K, Rackwitz B, Kober U, Wilke S, Ewert T, et al. Reliability of a test measuring transversus abdominis muscle recruitment with a pressure biofeedback unit. Physiotherapy. 2009 Mar; 95(1): 8-14.

[11] Malátová R, Pučelík J, Rokytová J, Kolář P. The objectification of therapeutical methods used for improvement of the deep stabilizing spinal system. 2007; 6 .

[12] Elgueta-Cancino E, Schabrun S, Danneels L, Hodges P. A clinical test of lumbopelvic control: development and reliability of a clinical test of dissociation of lumbopelvic and thoracolumbar motion. Man Ther. 2014 Oct; 19(5): 418-24.

[13] Kobesova A, Davidek P, Morris CE, Andel R, Maxwell M, Oplatkova L, et al. Functional postural-stabilization tests according to dynamic neuromuscular stabilization approach: proposal of novel examination protocol. J Bodyw Mov Ther. 2020 Jul; 24(3): 84-95.

[14] Chaitow L, Bradley D, Gilbert C. Recognizing and treating breathing disorders: a multidisciplinary approach. Edinburgh: Churchill Livingstone; 2014.

[15] Kobesova A, Safarova M, Kolar P. Dynamic neuromuscular stabilization: exercise in developmental positions to achieve spinal stability and functional joint centration. In: Hutson M, Ward A, eds. Oxford Textbook of Musculoskeletal Medicine [Internet]. Oxford University Press; 2015 [cited 2020 Jul 10]. pp. 678-89. Available from: http://oxfordmedicine.com/view/ $10.1093 / \mathrm{med} / 9780199674107.001 .0001 / \mathrm{med}-978019967410$ 7-chapter-61

[16] Liebenson C. Rehabilitation of the spine a patient-centered approach [Internet]. 2020 [cited 2020 Jul 10]. Available from: 
http://ovidsp.ovid.com/ovidweb.cgi?T=JS\&CSC=Y\&NEWS $=$ N\&PAGE=booktext\&D=books3\&AN=01996161/3rd_Edition .

[17] Casas E, Justes A, Calvo C. Exercises in motor development positions. what happens with the activity of antagonist muscle pairs? Pilot study. J Sport Rehabil [Internet]. 2019 Jan 1 [cited $2020 \mathrm{Jul}$ 10]; 28(1). Available from: https://journals.humankin etics.com/view/journals/jsr/28/1/article-jsr.2017-0389.xml. $\mathrm{xml}$.

[18] Mohammad Rahimi N, Mahdavinezhad R, Attarzadeh Hosseini SR, Negahban H. Effect of Dynamic Neuromuscular Stabilization Breathing Exercises on Some Spirometry Indices of Sedentary Students With Poor Posture. Phys Treat Specif Phys Ther J. 2019 Nov 30; 169-76.

[19] Kobesova A, Andel R, Cizkova K, Kolar P, Kriz J. Can Exercise Targeting Mid-Thoracic Spine Segmental Movement Reduce Back Pain and Improve Sensory Perception in CrossCountry Skiers? Clin J Sport Med [Internet]. 2018 Nov 14 [cited 2020 Jul 10]; Publish Ahead of Print. Available from: https://journals.lww.com/00042752-900000000-99097.

[20] Cha YJ, Yoon H, Jung DH, Hwang J, You J, Sung H. The best lumbothoracic-cervical chain stabilization exercise for longus colli activation. J Med Imaging Health Inform. 2018 Jan 1; 8(1): 84-7.

[21] Kobesova A, Dzvonik J, Kolar P, Sardina A, Andel R. Effects of shoulder girdle dynamic stabilization exercise on hand muscle strength. Isokinet Exerc Sci. 2015 Mar 1; 23(1): 21-32.

[22] Kim DH, Lee JJ, You S, Joshua H. Best core stabilization exercise to facilitate subcortical neuroplasticity: a functional MRI neuroimaging study. Technol Health Care. 2018 Jun 29; 26(3): 401-7.

[23] Scott J, Huskisson EC. Graphic representation of pain. Pain. 1976 Jun; 2(2): 175-84

[24] Jebavy R, Baláš J, Vomackova H, Szarzec J, Stastny P. The effect of traditional and stabilization-oriented exercises on deep stabilization system function in elite futsal players. Sports. 2020 Nov 28; 8(12): 153.

[25] Akoglu H. User's guide to correlation coefficients. Turk J Emerg Med. 2018 Sep; 18(3): 91-3.

[26] Ivanenko Y, Gurfinkel VS. Human postural control. Front Neurosci. 2018 Mar 20; 12: 171.

[27] Bradley H, Esformes J. Breathing pattern disorders and functional movement. Int J Sports Phys Ther. 2014 Feb; 9(1): 28 39.

[28] Kang J-I, Jeong D-K, Choi H. Effect of exhalation exercise on trunk muscle activity and oswestry disability index of patients with chronic low back pain. J Phys Ther Sci. 2016; 28(6): 1738-42.
[29] Park S-H, Lee M-M. Effects of a progressive stabilization exercise program using respiratory resistance for patients with lumbar instability: a randomized controlled trial. Med Sci Monit. 2019 Mar 7; 25: 1740-8.

[30] Jung S, Hwang U, Ahn S, Kim H, Kim J, Kwon O. Lumbopelvic motor control function between patients with chronic low back pain and healthy controls: a useful distinguishing tool. Medicine (Baltimore). 2020 Apr; 99(15): e19621.

[31] Huxel Bliven KC, Anderson BE. Core stability training for injury prevention. Sports Health Multidiscip Approach. 2013 Nov; 5(6): 514-22.

[32] Iqbal K. Mechanisms and models of postural stability and control. In: 2011 Annual International Conference of the IEEE Engineering in Medicine and Biology Society [Internet]. Boston, MA: IEEE; 2011 [cited 2020 Jul 5]. pp. 7837-40. Available from: http://ieeexplore.ieee.org/document/6091931/.

[33] Kuo AD. An optimal control model for analyzing human postural balance. IEEE Trans Biomed Eng. 1995 Jan; 42(1): 87101.

[34] Norris CM. Back stability: integrating science and therapy. 2nd ed. Champaign, IL: Human Kinetics; 2008; 347.

[35] Safarova M, Alena K, Pavel K. Dynamic neuromuscular stabilization and the role of central nervous system control in the pathogenesis of musculoskeletal disorders. In: Hutson M, Ward A, eds. Oxford Textbook of Musculoskeletal Medicine [Internet]. Oxford University Press; 2015 [cited 2020 Jul 5]. pp. 66-83. Available from: http://oxfordmedicine.com/view/ 10.1093/med/9780199674107.001.0001/med-978019967410 7-chapter-8.

[36] Garcia AN, Costa L da CM, de Souza FS, de Almeida MO, Araujo AC, Hancock M, et al. Reliability of the mechanical diagnosis and therapy system in patients with spinal pain: a systematic review. J Orthop Sports Phys Ther. 2018 Dec; 48(12): 923-33.

[37] Lima PO de P, de Oliveira RR, Costa LOP, Laurentino GEC. Measurement properties of the pressure biofeedback unit in the evaluation of transversus abdominis muscle activity: a systematic review. Physiotherapy. 2011 Jun; 97(2): 100-6.

[38] Cha YJ, Lee JJ, Kim DH, You J (Sung) H. The validity and reliability of a dynamic neuromuscular stabilization-heel sliding test for core stability. Technol Health Care. 2017 Oct 23; 25(5): 981-8.

[39] Laufer S, Pugh CM, Van Veen BD. Modeling touch and palpation using autoregressive models. IEEE Trans Biomed Eng. $2018 \mathrm{Jul}$; 65(7): 1585-94.

[40] Lawrance SE, Voll CA, Jochum JE. Enhancing palpation skills through the use of stereognosis drills. Athl Train Educ J. 2016 Jul 1; 11(3): 146-51. 